\title{
Self-Inclusion of Proline-Functionalised Calix[4]arene Leads to Hydrogelation
}

\author{
Ching Yong Goh, ${ }^{a}$ Thomas Becker, ${ }^{a}$ David H. Brown, ${ }^{a}$ Brian W. Skelton, ${ }^{b}$ Franca Jones, ${ }^{a}$ Mauro \\ Mocerino $^{* a}$ and Mark I. Ogden ${ }^{* a}$
}

5 Received (in $X X X, X X X) X$ th $X X X X X X X X X 20 X X$, Accepted $X$ th $X X X X X X X X X 20 X X$ DOI: 10.1039/b000000x

Solution-phase and solid-state structural studies indicate that the remarkable hydrogelation properties of a prolinefunctionalised calix[4] arene emerge as a result of extended

10 helical structures formed via inclusion of a proline moiety in a neighbouring calixarene cavity.

The majority of reported examples of gelators involve polymeric systems. More recently, low molecular weight gelators have been of interest, because the supramolecular interactions that cause

15 gelation are reversible, and can respond to specific stimuli, opening up a range of possible applications. ${ }^{1-3}$ The relatively simple structure of the molecular gelator, compared to a polymer, also makes design of the system a more tractable, but still a very challenging problem, ${ }^{4}$ with the aim being to drive the anisotropic

20 aggregation required to form the fibrous structures observed in many gels. ${ }^{5}$ The properties of the resulting gels can be readily tuned, particularly when using multi-component systems, ${ }^{4,6-9}$ or by adding or changing additives such as simple ions. ${ }^{10-14}$ We have previously described the electrolyte-triggered hydrogelation

25 behaviour of the first calixarene-based hydrogelator, prolinefunctionalised calix[4]arene $1 .{ }^{15}$ While we were unable to propose a detailed mechanism for the anisotropic aggregation of this system, it was shown that the gel formation was controlled by both electrolyte concentration and composition, as well as $\mathrm{pH}$.

30 Zhang et al. very recently reported that $\mathbf{1}$ will also form hydrogels in the presence of basic amino acids under neutral to acidic conditions. ${ }^{16}$ We now report a possible mechanism of assembly that provides the structural information required to refine the properties of this unique hydrogelator. We also demonstrate that

35 the chirality of the molecule has a direct impact on the bulk properties of the hydrogel formed.

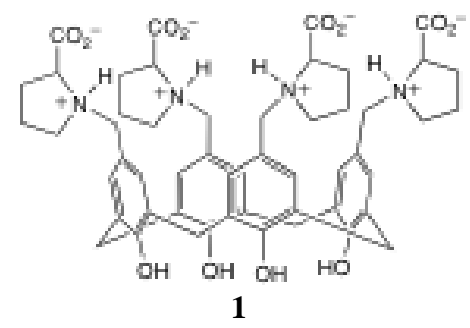

The impact of chirality upon gelation processes has been 40 reviewed recently. ${ }^{17}$ To probe the importance of chirality in $\mathbf{1}$, calix[4]arene was functionalised with D-proline, following the previously reported synthetic procedure. ${ }^{15}$ As expected the gelation properties were found to be identical to those of the Lproline calix[4]arene. Racemic mixtures, in contrast, were found 45 to have lower $\mathrm{T}_{\text {gel }}$ values, consistent with observations reported previously for dendritic peptides, ${ }^{18}$ and other (but not all ${ }^{17}$ ) racemates, ${ }^{19}$ reaching a minimum at the $1: 1$ ratio of $\mathrm{D}$ and $\mathrm{L}$ isomers (Figure $\mathrm{S} 1$ ). The reduction in $\mathrm{T}_{\text {gel }}$ was found to be $\sim 10{ }^{\circ} \mathrm{C}$ for gels formed by the addition of lanthanum nitrate, by both the 50 dropping ball, and flask inversion methods. ${ }^{\dagger}$ Atomic force microscopy of the wet gels, on mica, revealed that the fibrous structure of the gels was significantly altered in the racemate, consistent with the changes observed in bulk properties (Figure 1). The racemate appears to form linked spheroidal structures, 55 whereas the pure chiral systems form more elongated fibrous structures.

(a)

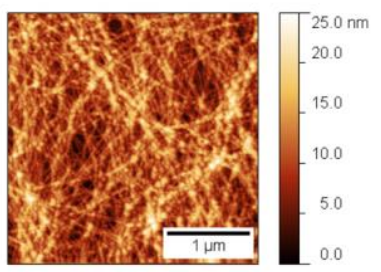

(b)

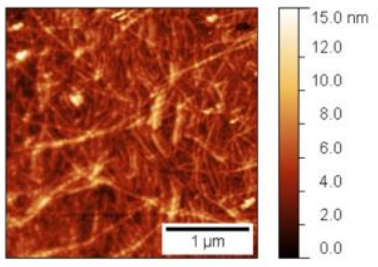

(c)

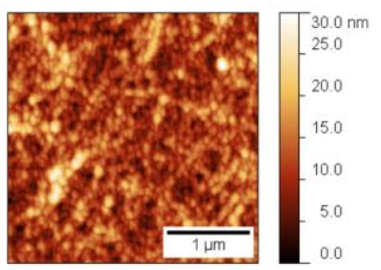

60 Fig. 1. Atomic force micrographs of $(\mathbf{1}+$ lanthanum nitrate $)$ hydrogels deposited as thin films on mica and imaged immediately. (a) L-isomer,

(b) D-isomer, and (c) DL-racemic mixture. 
Racemic mixtures are also reported to crystallise more readily than the pure enantiomers, in some cases not forming gels at all. ${ }^{18}$ In the present case, the racemic gels were found to be less stable

5 than the enantiomerically pure systems, tending to collapse and crystallise over a period of days, rather than remaining stable for months as in the case of the enantiomerically pure samples. The crystals that formed in one case were of sufficient quality to carry out an X-ray structure determination. ${ }^{\dagger, *}$

10 The resulting X-ray structure provides a fascinating insight into possible structural features of the fibrous network formed by this molecule. The previously reported structure of L-1 showed a typical layered structure, with the calixarene cavity occupied by two 1,3-disposed proline substituents, turning 'inwards' in such a

15 way that the carboxylate $\mathrm{O}$ atoms were at a distance $(2.47 \AA)$ consistent with a strong intramolecular hydrogen bond. ${ }^{16}$ This structure did not, however, provide any indication of how anisotropic assembly might develop. In contrast, the present structure shows intermolecular inclusion of a proline moiety into

20 the cavity of a neighbouring calixarene which results in the formation of a helical structure (Fig. 2). Each helical structure is formed by a single enantiomer, either the D- or L-isomer, indicating that the individual enantiomers could form comparable structures, as the fundamental unit from which larger fibres, and

25 fibre bundles, are formed.

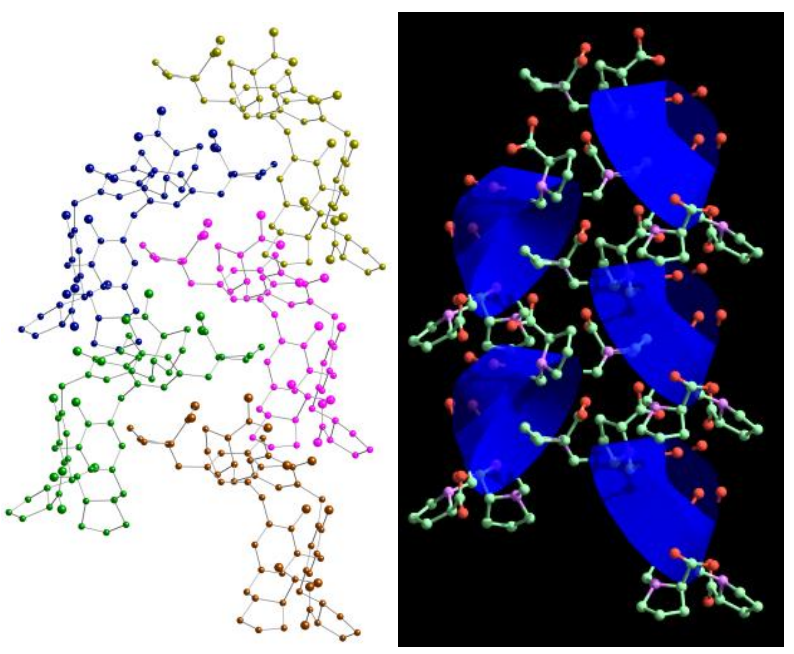

Fig. 2. The helical packing of $\mathbf{1}$ found in the La(DL-1) crystal structure; a ball and stick model coloured by molecule, and a schematic diagram with the calixarene backbone represented by blue ribbons. ESI includes an animation of the ribbon representation. $\dagger$

Looking at the structure in more detail, the lanthanum cations bridge two supramolecular helices to form a 2-dimensional coordination polymer. The $\mathrm{La}$ atom is bonded to both the carboxylate oxygen atoms of proline 24 and also to both of these

35 atoms of the centrosymmetrically related molecule (Fig. 3). The $\mathrm{La}$ atom is also bonded to the carboxylate oxygen $\mathrm{O} 141$ of the molecule related by a cell translation along the $b$ direction. The polymeric sheets are packed in the unit cell with the proline group (4) of the sheet inserted into the calixarene cavity related

40 by a 2 -fold screw axis (Fig. 4). A key feature is that the La ions are bridging two helices, each of which are formed from only one of the isomers, i.e. the La bridges a helix of D-1 with a helix of L1.

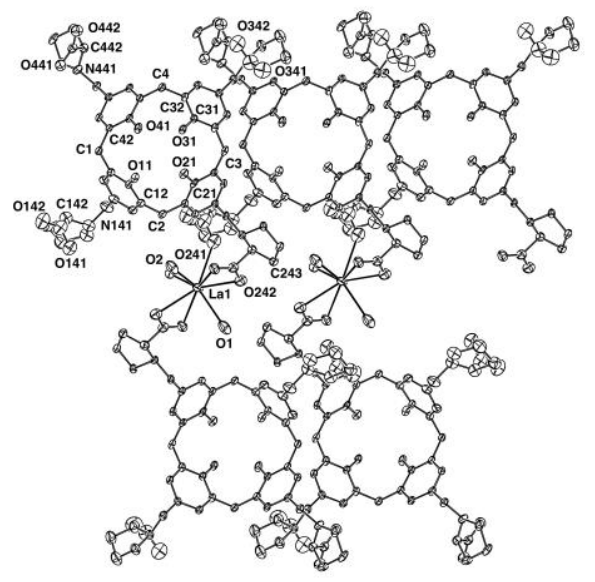

Fig. 3. The structure of La(DL-1) projected along the pseudo 4-fold axis of the calixarene showing the coordination polymer and the coordination around the La1 atom.

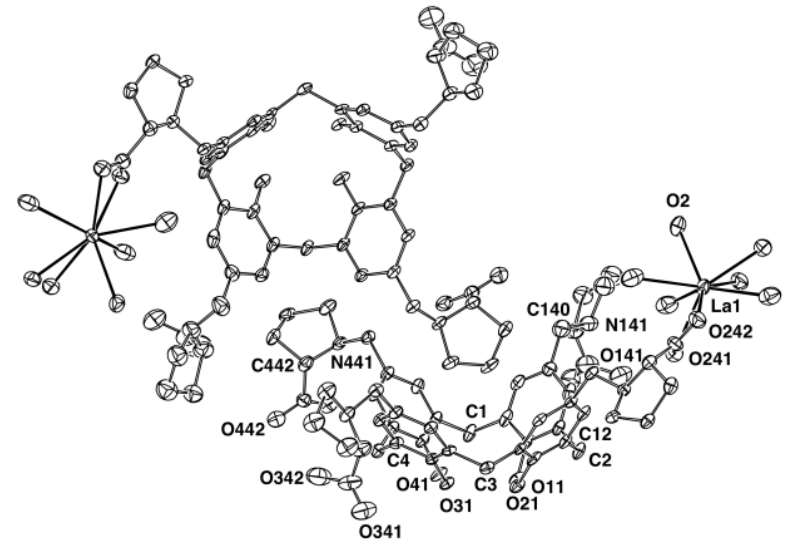

Fig. 4 The structure of La(DL-1) showing the proline moiety of the symmetrically related molecule inserted into the calixarene cavity.

It is notable that the lanthanum atom position appears to be not fully occupied, and that no nitrate anions could be located in the lattice. This causes some difficulty in determining the protonation 55 state of both calixarene and coordinated and free water molecules. It may be that charge balance is achieved by deprotonation at $\mathrm{N} 441^{*}$ and/or water molecules. The presence of a highly disordered nitrate anion that could not be specifically modelled, however, cannot be ruled out.

60 Proton NMR studies of $\mathbf{1}$ alone in $\mathrm{D}_{2} \mathrm{O}$ show broadening as a function of concentration, suggesting that the strands observed in the solid-state may be forming spontaneously in solution as the concentration is increased (Figure S2).$^{\dagger}$ These structures are thermally reversible, shown through a high temperature VT-NMR 65 study (Figure S3). Introduction of lanthanum nitrate results in peak broadening at lower concentrations, consistent with the electrolyte-triggered hydrogelation reported previously (Figure S4). ${ }^{15}$ It is notable that less than an equivalent of $\mathrm{La}$ is needed to result in significant broadening of the NMR spectra (Figure S5).

70 While the nitrate anion could not be located, or was not present, in the lattice, the structure reported here nevertheless 
provides critical information that allows us to propose a gelation mechanism for this system. Our previous work showed a strong relationship with the anion introduced as the electrolyte trigger. Nitrate favoured gelation strongly, for example, whereas sulfate

5 salts did not form a gel under any conditions. ${ }^{15}$ This behaviour was suggested to be related to the Hofmeister or Common Ion Effect. ${ }^{20}$ The nature of the cation had a moderating influence on the gel formation process. The results reported here lead us to propose a gelation mechanism that commences with spontaneous

10 assembly of $\mathbf{1}$ in water as helical strands. Added metal cations may then link these strands together to form more robust fibres. Gels formed in the presence of acids ${ }^{15}$ or organic cations ${ }^{16}$ may involve similar structures linked by hydrogen bonds. Finally, the anion present plays the dominant role in determining how these

15 fibres aggregate to form extended bundles, and ultimately a network that stabilises a gel.

\section{Conclusions}

A possible mechanism of hydrogelation for the unique gelator $\mathbf{1}$ has been proposed, based on solution and solid-state studies. The

20 results obtained will enable further structural development and diversification of this class of hydrogelator, and suggests ways to disrupt and/or control the gel formed (by adding hydrophobic guests for example). Further work is underway studying the solution-phase behaviour in more detail, as well as

25 characterisation of the gels using SAXS and cryo-TEM to complement the atomic force microscopy results.

\section{Notes and references}

${ }^{a}$ Nanochemistry Research Institute, Department of Chemistry, Curtin University, GPO Box U 1987, Perth, Australia 6845. Fax: +6189266

30 4699; Tel: +6189266 3838; E-mail: m.mocerino@curtin.edu.au, m.ogden@curtin.edu.au

${ }^{b}$ School of Biomedical, Biomolecular and Chemical Sciences, University of Western Australia, Crawley 6009 WA, Australia.

$35 \uparrow$ Electronic Supplementary Information (ESI) available; X-ray structural data of $\mathrm{La}(\mathrm{DL}-1)$; $\quad$ CCDC 815038 contains the supplementary crystallographic data for this paper. These data can be obtained free of charge from The Cambridge Crystallographic Data Centre. See DOI: $10.1039 / \mathrm{b} 000000 \mathrm{x} /$

40 ‡ Crystal data for $\mathrm{La}(\mathrm{DL}-1) ; \mathrm{C}_{52} \mathrm{H}_{73.60} \mathrm{La}_{0.40} \mathrm{~N}_{4} \mathrm{O}_{18.80}, M=1111.11$, monoclinic, space group $C 2 / c, \mathrm{Z}=8, a=35.5856(13), b=9.6028(3), c$ $=35.8812(9) \AA, \beta=99.056(3)^{\circ}, V=12108.5(7) \AA^{3}, \mu=0.363 \mathrm{~mm}^{-1}, \rho_{\mathrm{c}}=$ $1.219 \mathrm{Mg} \mathrm{m}^{-3}, \mathrm{~T}=100(2) \mathrm{K}$. Absorption $\mathrm{T}_{\max . / \min .}=1.00 / 0.81, \theta_{\max }=$ $25.00^{\circ}$. Total reflections 22605 , unique $10435\left(\mathrm{R}_{\text {int }}=0.050\right), R_{1}=0.112$

$45(I>2 \sigma(I)), w R_{2}=0.374$ (all data). La1 was also modelled as being disordered about an inversion centre with site occupancy refining to 0.385 (2) (see also Table $\mathrm{S}^{\dagger}$ ). Two coordinated water molecules were assigned occupancies constrained to that of the La1 atom. Hydrogen atoms were located on three proline $\mathrm{N}$ atoms. Since no hydrogen atom

50 was located on N441 nor did there appear to be an associated water molecule suitable for hydrogen bonding, this hydrogen atom was not included in the model. The program Squeeze ${ }^{21}$ was used to effectively remove electron density which could not be modelled as solvent or anions. See Tables S1 and S2 for more details.

55 1. D. K. Smith, Molecular Gels - Nanostructured Soft Materials, in Organic Nanostructures, eds. J. L. Atwood and J. W. Steed, WileyVCH, Weinheim, 2008, pp. 111-154.

2. R. G. Weiss and P. Terech, eds., Molecular Gels, Springer, Dordrecht, 2006.

60 3. J. W. Steed, Chem. Commun., 2011, 47, 1379-1383.
4. A. R. Hirst, I. A. Coates, T. R. Boucheteau, J. F. Miravet, B. Escuder, V. Castelletto, I. W. Hamley and D. K. Smith, J. Am. Chem. Soc., 2008, 130, 9113-9121.

5. L. A. Estroff and A. D. Hamilton, Chem. Rev., 2004, 104, 1201-1217.

65 6. L. Frkanec and M. Zinic, Chem. Commun., 2010, 46, 522-537.

7. A. R. Hirst and D. K. Smith, Chem. Eur. J., 2005, 11, 5496-5508.

8. A. Saha, S. Manna and A. K. Nandi, Langmuir, 2007, 23, 1312613135.

9. X. F. Zhu, Y. G. Li, P. F. Duan and M. H. Liu, Chem. Eur. J., 2010, 16, 8034-8040.

10. A. Brizard, C. Aime, T. Labrot, I. Huc, D. Berthier, F. Artzner, B. Desbat and R. Oda, J. Am. Chem. Soc., 2007, 129, 3754-3762.

11. J. A. Foster, M. O. M. Piepenbrock, G. O. Lloyd, N. Clarke, J. A. K. Howard and J. W. Steed, Nat. Chem., 2010, 2, 1037-1043.

75 12. G. O. Lloyd and J. W. Steed, Nat. Chem., 2009, 1, 437-442.

13. G. O. Lloyd and J. W. Steed, Soft Matter, 2011, 7, 75-84.

14. J. W. Steed, Chem. Soc. Rev., 2010, 39, 3686-3699.

15. T. Becker, C. Y. Goh, F. Jones, M. J. McIldowie, M. Mocerino and M. I. Ogden, Chem. Commun., 2008, 3900-3902.

80 16. J. Zhang, D.-S. Guo, L.-H. Wang, Z. Wang and Y. Liu, Soft Matter, 2011, 7, 1756-1762.

17. D. K. Smith, Chem. Soc. Rev., 2009, 38, 684-694.

18. A. R. Hirst, D. K. Smith, M. C. Feiters and H. P. M. Geurts, Chem. Eur. J., 2004, 10, 5901-5910.

85 19. A. Brizard, R. Oda and I. Huc, Top. Curr. Chem., 2005, 256, $167-$ 218.

20. W. Kunz, Curr. Opin. Colloid Interface Sci., 2010, 15, 34-39.

21. A. L. Spek, J. Appl. Crystallogr., 2003, 36, 7-13. 